\title{
Eficacia de la Constitución y derechos sociales: esbozo de algunos problemas
}

Desde hace décadas, la teoría constitucional tiene entre sus problemas más importantes el tema de la eficacia de sus normas. Aunque se trata de una cuestión que afecta, en general, a todas las normas constitucionales, el problema se hace más agudo tratándose de los derechos sociales.

En las páginas que siguen se ofrece una explicación de las dificultades que enfrentamos al intentar hacer eficaces las normas constitucionales a partir de una lectura histórica y conceptual de los derechos sociales y del modelo de Estado que los puede hacer realidad: el Estado social.

\section{Fundamento, justificación y antecedentes históricos de los derechos sociales: el modelo del Estado social}

Cualquier análisis sobre los derechos sociales debe tomar en cuenta o partir de la base de que, para poder ser realizados en la práctica, tales derechos requieren de un cierto modelo de organización estatal ${ }^{1}$, de una serie de precondiciones de carácter psicológico y de una base axiológica que permita reconocer el deber moral de hacernos cargo de las necesidades de los demás. Ninguna de las tres cuestiones mencionadas se encontraba presente en el primer constitucionalismo, en parte debido a que no lo permitían sus antecedentes históricos más inmediatos. El surgimiento y consolidación de los derechos sociales, con todos los problemas y limitaciones que se quiera, es un aspecto propio de los ordenamientos jurídicos desarrollados, aquellos que han acogido -al menos como ideal-al paradigma del neoconstitucionalismo ${ }^{2}$.

* Profesor de Derecho Constitucional e Investigador del lnstituto de Investigaciones Juridicas, Universidad Nacional de México $(U N A M)$
Gerardo Pisarello senala, con razón, que "Desde su irrupción como categoria histórica y teórica, la suerte de los derechos sociales ha estado anclada a la del propio Estado". "I)el Estado social tradicional al Estado social constirucional: por una protección compleja de los derechos sociales" en Carbonell, Miguel (compilador), Teoría constitucional y derechos fundamentales, México, CNDH, 2002, p. 115.

Carboncll, Miguel (edicor), Neoconstitucionalismo(s), 3 edición, Madrid, Trotta. 2006; id. Teoria del neoconstitu- 
Los presupuestos necesarios para dotar de eficacia práctica a los derechos sociales y a las normas constitucionales que los contienen se pueden agrupar conceptualmente en la noción de "Estado social", dado que su nacimiento, desarrollo y expansión es una condición esencial para la existencia de los propios derechos sociales ${ }^{3}$.

Para explicar el surgimiento y la crisis del Estado social hay muchas teorías, cada una de las cuales emplea un diferente método de análisis y encuentra una determinada forma de justificación ${ }^{4}$. Es muy posible que a varias les asista parte de razón, pero que ninguna pueda reclamar para sí el monopolio de la verdad en esta materia; en el estudio de procesos históricos marcados por una gran complejidad, que se desarrollan de forma desigual en cada país y que están sujetos a avances y retrocesos, quizá sea más útil tomar perspectivas de varias aproximaciones teóricas, más que centrarse en algún punto de vista; esto es lo que se hará en las páginas siguientes.

Las principales justificaciones del surgimiento del Estado social pueden dividirse, siguiendo a Contreras Peláez, en pluralistas y marxistas 5 .

Las justificaciones pluralistas pueden ser divididas, a su vez, en funcionalistas y conflictualistas. Para las primeras el Estado social surge como respuesta a necesidades objetivas suscitadas por la modernización socio-económica; es decir, en la medida en que el sistema económico va evolucionando y los trabajadores se especializan y emigran a las ciudades, el sistema político debe responder a través de la generación de los contenidos característicos del Estado social. El punto de vista conflictualista defiende que el Estado social es el producto de la presión política de los sectores más desfavorecidos, que a su vez es generada por la ampliación del sufragio y por el ejercicio del derecho de asociación, que permite una acción más eficaz de la clase obrera a través de los sindicatos.

Por su lado, las justificaciones marxistas también pueden ser divididas en funcionalistas y conflictualistas. Para las primeras el Estado social es la respuesta a ciertas exigencias estructurales del capitalismo maduro ${ }^{6}$; entre esas exigencias se encontraba, por ejemplo, la necesidad de asegurar la reproducción de la fuerza de trabajo, la necesidad de integrar al movimiento obrero dentro del sistema, abortando cualquier intento de acudir a la vía revolucionaria, etcétera. Para las visiones marxistas conflictualistas el Estado social sería el resultado de una serie de conquistas del proletariado y constituiría un episodio intermedio en la lucha de clases.

cionalismo. Ensayos escogidos, Madrid, Trotta, 2007.

3 Hay que señalar. sin embargo, que la idea de los "derechos sociales" como derechos de tango constitucional es previa al surgimiento del Estado social. Lo que sucede es que dicha idea no puede ser llevada a la práctica de forma completa y coherente justamente por la falta de las estructuras estatales que lo permitan; el surgimiento del Estado social vendrá a significar, entonces, la posibilidad de realizar en la práctica esa idea de los derechos sociales como derechos que deben ser tutelados por el sistema constitucional. Al respecto, Böckenförde, Ernst-Wolfgang, Escritos sobre derechos fundumentales, Baden-Baden, Nomos, 1993, pp. 72 y ss.

4 Además de las obras que se mencionan en las siguientes notas, un buen panorama introductorio puede ver en Esping-Andersen, Gosta, Fundamentos sociales de las economias postindustriales, Barcelona, Ariel, 2000.

Contreras Peláez, Francisco José, Defensa del Estado social, Sevilla, Universidad de Sevilla, 1996, p. 12.

Sobre este tema habia insistido, desde sus primeros trabajos, Jürgen Habermas; por ejemplo en su libro Problemas de legitimación del capitalismo tardio, Buenos Aires, Amorrortu editores, 1975. 
Como quiera que sea, el surgimiento del Estado social se da en un contexto histórico en el que están presentes las siguientes tres condiciones ${ }^{7}$ :

A) El individuo es incapaz de satisfacer por sí solo, o con la ayuda de su entorno social más inmediato, sus necesidades básicas;

B) Surgen riesgos sociales que no pueden ser enfrentados por las vías tradicionales, basadas en la responsabilidad individual;

C) Se desarrolla la convicción social de que el Estado debe asumir la responsabilidad de garantizar a todos los ciudadanos un mínimo de bienestar; si el Estado no cumpliera con esa obligación, se pondría en duda su legitimidad.

Al tratarse de condiciones, por decirlo de alguna manera, estructurales para el desarrollo del Estado social, conviene detenernos brevemente en cada uno de los tres incisos que se acaban de enunciar.

\section{El individuo indefenso}

En las sociedades primitivas, y aun en las sociedades rurales pre-modernas, los individuos seguramente estaban tanto o más indefensos que los habitantes de nuestras sociedades contemporáneas. En los tiempos recientes esa indefensión ha tomado relevancia debido a: a) el cambio en las condiciones físicas o biológicas de nuestra vida social; b) la modificación de la estructura económica relacionada con las condiciones de trabajo; y c) un replanteamiento de las redes de asistencia social. Vamos por partes.

Respecto a las condiciones biológicas, hay que constatar que las personas hoy en día pueden vivir muchos más años que hace unos siglos; esto constituye un avance en toda regla y es uno de los signos más positivos de la modernidad, logrado en buena medida por el desarrollo de las ciencias médicas. Sin embargo, ocurre que al vivir más tiempo, se multiplican las "situaciones de dependencia" y aumenta el porcentaje de individuos que se encuentran en tales situaciones ${ }^{8}$. La vejez, la invalidez y las enfermedades crónicas son fenómenos de nuestro tiempo, que no existían prácticamente en las sociedades antiguas.

Además de eso, el desarrollo de las formas de trabajo y el propio desarrollo tecnológico han propiciado el surgimiento de situaciones "artificiales" de dependencia, como el desempleo, la jubilación obligatoria o el alargamiento de los periodos de formación antes de acceder al mercado de trabajo".

Conforme avanza el proceso de urbanización de las sociedades, las personas se vuelven más indefensas, ya que son incapaces de proveerse por sí mismas de los bienes básicos. Mientras que en las sociedades rurales abundaban los esquemas de auto-abastecimiento

Contreras Peláez, Francisco José, Defensa del Estado social, cit., p. 13.

Contreras Peláez, Francisco José, Defensa del Estado social, cit., p. 13.

Contreras Peláez, Francisco José, Defensa del Estado social, cit., p. 14. 
en el seno de las familias o de comunidades un poco más amplias, en la vida urbana es casi imposible que una familia pueda auto-generar su propia comida, su ropa, su vivienda y así por el estilo. Se requiere de la ayuda exterior, ya que el habitante de las ciudades es "un ser del todo desamparado"

Hay una gran diferencia en el sujeto de los derechos sociales propio del siglo XX y la imagen del trabajador del siglo XIX, que tenía una escasa o nula cualificación, que recién había emigrado del campo a la ciudad, que tenía una familia numerosa al no haberse desarrollado los métodos de control de la natalidad y que carecía de la protección de las reglas que luego iban a desarrollarse en materia de ingreso y estabilidad laboral"

Bajo el nuevo esquema al que me estoy refiriendo, las personas ya no dominan su espacio vital, aunque gozan de una mayor amplitud para realizar sus planes de vida; una amplitud propiciada en primer lugar por el alargamiento de los años de vida, y en segundo término por los desarrollos tecnológicos que han puesto a su disposición una serie de comodidades que hasta hace poco eran impensables. Pero ese nuevo entorno no es un entorno que dominen, sino que está determinado por la concurrencia de otras personas y por la prestación de un sinnúmero de servicios esenciales para la sociedad en su conjunto. Hoy como nunca los unos dependemos de los otros y no solamente de nuestro entorno más inmediato como lo era antes la familia o la gente de nuestra comunidad más inmediata.

Tanto las nuevas condiciones biológicas como las que tienen que ver con la forma de organización laboral desbordan las rutas tradicionales de asistencia social, basadas en la caridad o en el apoyo de la familia, y requieren necesariamente de apoyos exteriores; estos apoyos, a su vez, deben ser institucionalizados para operar eficientemente y gozar de la amplitud necesaria que les permita abarcar a grandes grupos sociales. Es entonces cuando el Estado comienza a hacerse cargo, desplazando de esa forma a las anteriormente conocidas formas de compasión y socorro Cristiános, y creando lo que se ha llamado la "burocratización de la compasión"12.

\section{Los nuevos riesgos sociales}

En las sociedades contemporáneas nos enfrentamos a riesgos que o no estaban presentes en las sociedades pre-modernas o bien no se consideraban relevantes para el ordenamiento jurídico, de forma que se dejaba a las personas libradas a su propia suerte.

Contreras Peláez recuerda al menos cuatro factores de vulnerabilidad que han generado respuestas jurídicas más o menos contundentes durante el siglo XX:

a) El desempleo, que ha producido el surgimiento de políticas económicas de pleno

Contreras Peláez, Francisco José, Defensa del Estado social, cit., p. 15-16.

Contreras Peláez, Francisco José, Defensa del Estado social, cit., p. 18.

Contreras Peláez, Francisco José, Defensa del Estado social, cit., p. 14. 
empleo, seguros contra el desempleo, redes de asistencia social e incluso de pensiones desligadas del carácter de trabajador que pueda o no tener el beneficiario, etcétera;

b) La crisis de La familia; el modelo actual de familia presenta muchas diferencias con respecto al modelo anterior; su nueva organización requiere de formas de auxilio externo, que permitan que se haga frente a los riesgos sociales colectivamente, sobre todo en el caso de familias monoparentales (un adulto viviendo con menores de edad), familias de ancianos, familias que tienen a su cargo personas con discapacidad, etcétera ${ }^{13}$;

c) el factor del sexo, que ha generado procesos de "feminización de la pobreza", que afecta principalmente a madres solteras, madres con familia numerosa y bajos ingresos y a ancianas que viven solas; $y$

d) la inmigración; en la actualidad, son los inmigrantes uno de los eslabones más débiles de las sociedades desarrolladas, pues en ellos se presentan, acentuadas, varias de las anteriores características: desempleo, desintegración familiar, exceso de cargas para la mujer, escasa preparación para el trabajo, dificultades de integración social, etcétera ${ }^{14}$.

Aparte de lo anterior, algunos desarrollos tecnológicos han contribuido también a generar nuevos riesgos sociales; esto se percibe claramente al analizar el aumento de los accidentes de trabajo o las enfermedades derivadas de riesgos laborales. La tecnología incide, a veces negativamente, en el disfrute de los derechos fundamentales ${ }^{15}$.

Tradicionalmente, la responsabilidad por los tiesgos personales era de carácter individual; es decir, quien causaba el daño era el obligado a repararlo: se trata del clásico esquema de la responsabilidad civil. Con el paso del tiempo y el aumento de los riesgos como consecuencia del desvalimiento del individuo, se consideró que era necesario cambiar este esquema para avanzar hacia una "socialización del riesgo", es decir, hacia la creación de mecanismos institucionales que fueran capaces de responder frente a los riesgos sociales.

Para tal efecto, el Estado debe actuar en dos frentes: la promoción del bienestar y la atenuación o compensación del sufrimiento ${ }^{16}$. Lo anterior conlleva la necesidad de que el Estado actúe frente al infortunio (accidentes de trabajo) y frente a la necesidad (seguro de desempleo, pensiones de viudez, por jubilación, por incapacidad, etcétera). De esta manera, surgen los que hoy conocemos como los modernos sistemas de seguridad social, que encuentran sus antecedentes en las "Leyes de Bismarck", dictadas a partir de

13 Beck-Gernsheim, Elisabech, La reinvención de la familia. En busca de nuevas formas de convivencia, Barcelona, Paidós, 2003.

14 Ver sobre el tema de la migración y los derechos fundamentales, Carbonell, Miguel, "Libertad de cránsito y fronteras: la gran cuestión del siglo XXI" en Valadés, Diego y Carbonell, Miguel (coordinadores), El proceso constituyente mexicano. A 150 años de la Constitución de 1857 y 90 de la Constitución de 1917, México, I1]-UNAM, 2007, pp. 103-124. Sobre la migración y las fronteras, puede verse el excelente ensayo de Kymlicka, Will, Fronteras territoriales. Madrid, Trotta, 2006.

is Doménech Pascual, Gabriel, Derechos fundamentales y riesgos tecnológicos, Madrid, CEPC, 2006.

16 Contreras Peláez, Francisco José, Defensa del Estado social, cit., p. 22. 
1883; entre ellas se encuentran la Ley de seguro de enfermedad y maternidad de 1883, la Ley de accidentes de trabajo de 1884 y la Ley de seguro de enfermedad, jubilación y defunción de $1889^{17}$.

La seguridad social, tanto por medio de la asistencia en caso de siniestro o la cobertura de riesgos (accidentes laborales, acceso a medicinas, etcétera), o bien a través de la cobertura de situaciones de necesidad (vejez, invalidez, etcétera) es una de las claves y de los signos distintivos del Estado social; quizá sea su nota más presente y representativa, pues a pesar de los varios modelos que existen de Estado social y de las diferentes configuraciones que cada uno de ellos puede tener en un contexto histórico o geográfico, lo que es obvio es que no puede hablarse de Estado social si no se cuenta con un sistema de seguridad social. Como lo señala Benda, la "Seguridad social es una expresiva traducción del postulado del Estado social"'8.

\section{El papel del Estado}

Los dos factores que se acaban de analizar (la mayor vulnerabilidad del individuo y la necesidad de hacer frente socialmente a los riesgos) exigen que se asuma un nuevo papel por parte del Estado. Esta es una de las transformaciones que en mayor medida van a afectar a la teoría de los derechos fundamentales y a los procesos de legitimación de los poderes públicos en relación con estos derechos.

Hay que recordar que, para el primer constitucionalismo, los derechos tenían que imponerse frente al Estado; es decir, los derechos se consideraban como una especie de valladar frente a las intromisiones de una estructura estatal que, antes de los movimientos revolucionarios de Francia y Estados Unidos, se conducía de manera despótica y no estaba sujeta a más límites que la voluntad del emperador, del rey o del caudillo ${ }^{19}$.

Sin embargo, en el modelo del Estado social, los poderes públicos dejan de ser percibidos como enemigos de los derechos fundamentales y comienzan a tomar, por el contrario, el papel de promotores de esos derechos, sobre todo de los de carácter social. Se entiende ahora que también la concentración de la riqueza y el avance tecnológico no sujetos a reglas pueden vulnerar los derechos fundamentales ${ }^{20}$.

De esta manera, la legitimidad de los poderes públicos no depende ya solamente de que no entorpezcan o limiten el disfrute de los derechos, sino también de que los promuevan eficazmente. Como lo señala Luigi Ferrajoli, en el Estado social se genera un cambio en los factores de legitimidad del Estado, pues

Ochendo Claramunt, Carlos, El Estado del bienestar. Objetivos, métodos y teorias explicativas. Barcelona, Arieł, 1999, p. 28.

is Benda, Ernesto, "El Estado social de derecho" en VV.AA., Manual de derecho constitucional, Madrid, Marcial Pons, 1996, p. 536.

Carbonell, Migue], Una historia de los derechos fundamentaler. México, Porrúa, UNAM, CNDH, 2005.

Fin esce scntido. Forsthoff, tinst. El Estudo de la mitedad industrial, Madrid, Instituto de Fistudios Políticos. 1975. 
“mientras el Estado de derecho liberal debe sólo no empeorar las condiciones de vida de los ciudadanos, el Estado de derecho social debe también mejorarlas, debe no sólo no representar para ellos un inconveniente, sino ser también una ventaja. Esta diferencia va unida a la diferente naturaleza de los bienes asegurados por los dos tipos de garantías. Las garantías liberales o negativas basadas en prohibiciones sirven para defender o conservar las condiciones naturales o pre-políticas de existencia: la vida, las libertades, las inmunidades frente a los abusos de poder, y hoy hay que añadir, la no nocividad del aire, del agua y en general del ambiente natural; las garantías sociales o positivas basadas en obligaciones permiten por el contrario pretender o adquirir condiciones sociales de vida: la subsistencia, el trabajo, la salud, la vivienda, la educación, etcétera. Las primeras están dirigidas hacia el pasado y tienen como tales una función conservadora; las segundas miran al futuro y tienen un alcance innovador" ${ }^{21}$.

Como ya se ha mencionado, los primeros derechos sociales - que todavía no alcanzan el rango de derechos fundamentales- surgen como formas de protección a los obreros, tanto frente a los infortunios derivados del ejercicio del trabajo, como frente a los patrones para regular las condiciones laborales de forma que no se permita el menoscabo de la dignidad humana.

Las reivindicaciones sociales se trasiadan al Estado, que comienza a incorporar entre sus funciones la de asistencia social. Sin embargo, los movimientos obreros insisten en que no se trata de sustituir la caridad privada por una caridad pública, sino de generar un conjunto de derechos que protejan a los trabajadores y a sus familias. La responsabilidad del Estado debe ser entendida, en consecuencia, como una responsabilidad juridica, garantizada incluso a nivel constitucional, de forma que la persona necesitada deje de ser objeto de la relación asistencial y se convierta en un sujeto portador de derechos ${ }^{22}$.

Se puede afirmar, intentando hacer una síntesis de algunas cuestiones que ya se han mencionado, que el Estado social se caracteriza por las siguientes notas ${ }^{23}$ :

a) Se constituye como un amplio pacto social, cuyos principales actores son el Estado, los trabajadores, las clases medias urbanas y los empresarios;

b) Busca atender las necesidades de grandes sectores sociales, principalmente a través de servicios de salud, vivienda y educación;

c) Cumple un papel estabilizador de la demanda interna mediante el impulso del consumo gubernamental;

d) Procura la paz social al institucionalizar vías reformistas, que sustituyen a las vías revolucionarias;

Derecho y razón, 5a edición, Madrid, Trotta, 2000, p. 862.

Contreras Peláez, Francisco José, Defensa del Estado social, cit., p. 41.

Carbonell, José, "Estado de bienestar" en VV.AA, Diccionario de denecho constitucional, 2a edición, México, LNAM Pornía, 2005, p. 229. 
e) Posibilita un marco de crecimiento económico continuo, sostenible y equilibrado;

f) Crea un régimen fiscal redistributivo; $y$

g) Constituye un amplio sector público, dotado de importantes funciones regulativas y, en algunos casos (de forma subsidiaria), directamente productivas.

\section{El Estado social como Estado constitucional}

Aunque cronológicamente las primeras disposiciones constitucionales en materia de derechos sociales se encuentran en las Constituciones de Querétaro en 1917 y de Weimar en $1919^{24}$, no es sino hasta la Ley Fundamental de Bonn, de 1949, cuando la fórmula del "Estado social de derecho" adquiere reconocimiento constitucional ${ }^{25}$.

En las primeras fórmulas de consagración de derechos sociales (así por ejemplo, en el caso de la Constitución mexicana ${ }^{26}$ ), más que el reconocimiento constitucional de una nueva forma de Estado, lo que se hacía era dar cobertura en el texto de la Carta Magna a los derechos de grupos sociales tradicionalmente marginados; así por ejemplo, trabajadores y campesinos, que eran grupos que habían alimentado los movimientos revolucionarios de las primeras décadas del siglo XX y que constituían la base social indispensable para la legitimación de los poderes públicos.

En cambio, en los textos de la segunda posguerra, dictados después de 1945, lo que se observa es la consagración constitucional de todo un entramado jurídico, un verdadero cambio de paradigma constitucional. Es entonces cuando podemos decir que el Estado social se consolida y, a partir de ese momento, comienza un importante proceso de expansión, tanto desde el punto de vista de sus contenidos como desde una perspectiva geográfica al incrementarse el número de países que intentan adaptarse a sus principios ${ }^{27}$.

No se trata solamente de que la Constituciones contengan solemnes manifestaciones de principio ("La República Federal de Alemania es un Estado federal democrático y social", dispuso el conocido artículo 20.1. de la Ley Fundamental de Bonn ${ }^{28}$; "Espańa se

Para una primera aproximación al surgimiento del constitucionalismo social en la Constitución de Weimar, ver Carmona Cuenca, Encarnación, El Estado Social de Derecho en la Constitución, cir., pp. 43 y ss.

25 Carmona Cuenca, El Estado Social de Derecho en la Constitución, cit., pp. 57 y $5 s$.

26 Carbonell, Miguel, Los derechos fundamentales en México, $2^{\text {a }}$ edición, México, Porrúa, UNAM, CNDH, 2006, capitulo cinco.

27 Cfr. Ochando Claramunt, Carlos, El Estado del bienestar. Objetivos, modelos y teorias explicativas, cit., pp. 32-33.

28 De la misma Constitución es interesante también ver el artículo 28. La importancia dada a la forma del Estado social recogida en el artículo 20 se refleja en la prohibición de que sea reformada, tal como lo dispone el articulo 79; estamos en presencia de lo que la teoría de la reforma constitucional denomina una "cláusula pétrea". Para una introducción al estudio de los derechos sociales en Alemania, de entre lo mucho que se ha escrito, puede verse Weber, Albrecht, "Estado social, derechos sociales fundamentales y prorección social en la República Federal de Alemania" en Muñoz Machado, Santiago y otros, Las estructuras del bienestar en Europa, Madrid, Civitas, Escuela Libre Editorial, 2000, pp. 569 y ss. 
constituye en un Estado social y democrático de Derecho, que propugna como valores superiores de su ordenamiento jurídico la libertad, la justicia, la igualdad y el pluralismo político", señala el artículo 1.1. de la Constitución española de 1978), sino que a ellas se les acompaña un conjunto de preceptos para hacer posible la intervención del Estado en la sociedad y para asignar a los poderes públicos las responsabilidades concretas que se derivan de la idea general según la cual deben tutelar la "procura existencial".

¿Qué cuadro del ordenamiento jurídico en general tenemos luego de la constitucionalización del Estado social? Siguiendo a Abramovich y Courtis, podemos sostener lo siguiente $^{29}$ :

A) Se introducen dimensiones colectivas en el derecho, lo que ocurre a través de la construcción de sujetos de derecho colectivos (sindicatos, grupos de consumidores, etcétera), a través de la articulación de instancias y facultades de negociación colectivas y con la construcción de categorías colectivas o grupales (la noción de medio ambiente, de grupo vulnerable o de salud pública).

B) Se toman en cuenta las desigualdades reales y no simplemente las formales; esto genera a su vez nuevas pautas interpretativas (como las que se expresan en las conocidas fórmulas favor operatori o favor consumatoris) y nuevas reglas procesales (por ejemplo en materia de carga probatoria, de presunciones en favor de partes procesalmente débiles, etcétera).

C) Se establecen límites a la autonomía de la voluntad; en áreas tradicionalmente dejadas al libre acuerdo de las partes entran en funcionamiento conceptos de orden público y de protección a la dignidad de las personas que pueden generar nulidades contractuales y un importante control estatal de la oferta de bienes públicos y servicios.

D) En relación con el inciso anterior, se limita el ámbito de actuación sujeto a mecanismos contractuales, regulados ahora - con las reglas del Estado social-por medio de leyes; esto produce un efecto de desmercantilización de algunos sectores como resultado del avance del derecho público sobre el derecho privado.

E) Se modifican los criterios de asignación de responsabilidades civiles, como consecuencia del cambio de óptica en materia de generación de riesgos (varios de los cuales se "socializan", para efecto de proteger a las personas más indefensas) y de distribución de costos.

F) Se amplían las funciones estatales; al asumir el Estado las tareas relacionadas con la realización efectiva de los derechos sociales, los órganos públicos se multiplican y la burocracia crece de forma sensible. El Estado no se limita a ejercer funciones regulatorias, sino que se reserva para sí ciertas áreas que se consideran estratégicas, lo que conlleva una ampliación de funciones y del gasto público.

29 Abramovich, Víctor y Courtis, Christian, Los derechos sociales como derechos exigibles, $2^{2}$ edición, Madrid, Trotta, 2004, pp. 54-56. 
G) Se incorporan al ordenamiento acciones procesales de carácter colectivo, a través de la ampliación en las posibilidades de acceso a los tribunales (acciones de cumplimiento, acciones de tutela, acciones de clase, etcétera) y por medio de la tutela de bienes colectivos.

En todo caso, importa en este momento subrayar que la constitucionalización del Estado social es una tendencia firme del constitucionalismo contemporáneo, que ha conllevado un cambio de paradigma para el constitucionalismo y para la democracia, quizá el más importante en el desarrollo del Estado constitucional en el siglo $\mathrm{XX}^{30}$, con el objetivo de proteger de mejor manera valores esenciales de las sociedades modernas; tales valores, bajo ese nuevo paradigma, adquieren una protección inédita en otros tiempos al ser reconocidos como derechos fundamentales.

\section{Hacia una visión normativa de los derechos sociales}

Los derechos sociales tienen que ser entendidos - dejando atrás las concepciones tradicionales de signo fuertemente conservador-como derechos plenamente exigibles ante todas las autoridades del Estado, en todos sus niveles de gobierno. La plena exigibilidad requiere de la creación de una sólida teoría de los derechos sociales, así como de la puesta en marcha de nuevos mecanismos procesales o del mejoramiento de los ya existentes.

Lo anterior significa que, por poner un ejemplo, el derecho a la vivienda genera obligaciones lo mismo para las administraciones públicas, que para los Congresos o parlamentos, o que el derecho a la salud debe ser también y en primer término resguardado por el legislador, de forma que en la ley se definan concretamente las obligaciones del Estado en la materia, así como las prerrogativas de los particulares frente a los órganos públicos para poder hacer efectivas dichas obligaciones. En idéntica situación se encuentran el resto de derechos sociales, los cuales despliegan posiciones subjetivas en favor de todas las personas, a la vez que generan obligaciones para los poderes públicos, en todos sus niveles.

Para poder desarrollar plenamente la normatividad de los derechos sociales hace falta trabajar -entre otras- en dos cuestiones concretas:

\subsection{El contenido de los derechos sociales}

La primera es la que consiste en determinar el contenido semántico y los alcances concretos de cada derecho social; así por ejemplo, se debe estar en capacidad de determinar qué significa específicamente que la Constitución mexicana establezca el derecho a una

\footnotetext{
- Como afirma José Carbonell, "Sin duda, puede afirmarse que el resultado más importante del proceso de modernización de la segunda mitad del siglo XX fue la irrupción del componente social dentro de los principios organizarivos y recrores de las democracias contemporáneas", "Estado de bienestar", cit., p. 231.
} 
vivienda "digna y decorosa"; ¿en qué consiste y qué alcances tiene la "dignidad" y el "decoro" de la vivienda?, ¿cuándo se viola ese mandato constitucional?, ¿qué debe hacer el Estado para darle cumplimiento?, ¿qué significa que la misma Constitución reconozca el derecho a un medio ambiente "adecuado"?, ¿cuándo el medio ambiente deja de ser adecuado?, ¿qué corresponde hacer a los particulares y qué a las autoridades para preservar el medio ambiente? Y así por el estilo para todos los derechos sociales.

Las dificultades de determinación del contenido de los derechos sociales sin duda que existen y sin duda que son un obstáculo que hay que superar para poder hacer plenamente normativos esos derechos, pero no hay que ver en ello un problema insuperable; puede decirse que lo mismo sucede con los derechos de libertad; ¿̨cómo entender los alcances del derecho a la intimidad?, ¿qué significa en concreto la libertad de procreación?, ¿cuáles son los límites de la libertad de expresión y cuáles las obligaciones del Estado para protegerla? La apertura semántica no puede significar, por sí sola, una pérdida de los efectos normativos que pueden derivar de los derechos sociales.

La determinación del campo semántico de los derechos sirve, entre otras cuestiones, para poder determinar las obligaciones mínimas de los poderes públicos en relación con cada derecho social ${ }^{31}$. Realizar dicha determinación es una tarea que corresponde desarrollar, en primer término, al legislador, que a través de las leyes debe determinar contenidos concretos para cada derecho ${ }^{32}$. También es una función de la ciencia jurídica, pues en la medida en que se avance en el plano teórico se podrá avanzar en el plano práctico.

De forma preliminar, hay que mencionar que el surgimiento de los derechos sociales representa un cambio profundo respecto a la concepción que sobre los derechos se tenía en el primer liberalismo y que supone también una modificación sustancial en relación al entendimiento del papel del Estado en materia de derechos fundamentales.

De ser entendidos como derechos de defensa, en la actualidad los derechos fundamentales pasan a ser derechos de participación democrática y también, como sucede con algunos aspectos de los derechos sociales, derechos a prestaciones suministradas por el Estado ${ }^{33}$. No

31 Para Abramovich y Courtis, la determinación de esas obligaciones mínimas es quizá "el principal déficit del derecho constitucional $y$ del derecho internacional de los derechos humanos, tanto en la formulación de las normas que consagran los derechos, cuanto en las elaboraciones de los órganos nacionales e internacionales encargados de la aplicación de cláusulas constitucionales o de tratados, y en los escasos aportes doctrinarios al repecto". Abramovich. Victor y Courtis, Christian, Los derechos sociales como derechos exigibles, cit., pp. 38-39.

32. Al respecto Luigi Ferrajoli, en una observación que se dirige también al tema de la exigibilidad procesal de los derechos sociales, señala que “... seria necesario que las leves en materia de servicios públicos no sólo establecieran contenidos y presupuestos de cada derecho social, sino que identificasen también a los sujetos de derecho público investidos de las correlativas obligaciones funcionales; que toda omisión o violación de tales obligaciones, al comportar la lesión no ya de meros deberes o a lo sumo de intereses legítimos sino directamente de derechos subjetivos, diera lugar a una acción judicial de posibie ejercicio por el ciudadano perjudicado; que la legitimación activa fuera ampliada, en ios derechos sociales de naturaleza colectiva, también a los nuevos sujecos colectivos, no necesariamente dotados de personalidad juridica, que se hacen portadores de los mismos; que, en suma, junto a la participación política en las actividades de gobierno sobre las cuestiones reservadas a la mayoria, se desarrollase una no menos importante y generalizada participación judicial de los ciudadanos en la tutela y la satisfacción de sus derechos como instrumento tanto de autodefensa cuanto de control en relación con los poderes públicos ". Derecho y razón, $5^{a}$ edición, Madrid, Trotta, 2000, p. 918.

33 González Moreno, Beatriz, El Estado social Naturaleza juridica y estructura de los derechos sociales, Madrid, Civitas, 
es posible plantear, en la materia que nos ocupa, puntos de vista ingenuos: los derechos sociales, para ser realizados, requieren de una cierta organización estatal, necesitan de un apoyo social, de un conjunto de actitudes cívicas y un compromiso democrático serio.

\subsection{La exigibilidad procesal}

La segunda cuestión en la que se tiene que trabajar en materia de derechos sociales es en la denuncia de la inexistencia, dentro de muchos ordenamientos jurídicos contemporáneos, de vías procesales idóneas para hacerlos exigibles, así como en la necesidad de crear esos medios de defensa, de forma que sus violaciones puedan ser llevadas ante los tribunales o ante otros órganos protectores de los derechos fundamentales.

Que esas vías no existan, como ya se ha dicho, no significa que los derechos sociales no obliguen de forma plena a los órganos públicos; implica simplemente, lo cual no es poco desde luego, que sus violaciones no podrán ser reparadas por medio de juicios llevados ante los tribunales nacionales. En este contexto, a la ciencia jurídica le corresponde el deber de sugerir vías alternativas a la de los tribunales para exigir los derechos sociales, así como proponer la creación de procedimientos de carácter judicial para subsanar la laguna que se genera a partir de su inexistencia.

Hay que señalar, sin embargo, que las vías procesales no agotan los medios de exigibilidad de los derechos fundamentales; aunque los expertos indican que el reconocimiento universal de los derechos sociales, económicos y culturales como derechos plenos no se alcanzará hasta superar los obstáculos que impiden su adecuada justiciabilidad, entendida como la posibilidad de reclamar ante un juez o tribunal de justicia el cumplimiento al menos de algunas de las obligaciones que se derivan del derecho, no hay que pensar que el poder judicial es la única vía para hacer exigibles esos derechos; hay otros mecanismos que pueden ser tanto o más eficaces ${ }^{34}$. Es importante considerar lo que se acaba de decir, sobre todo en el caso de México y de otros países de América Latina, puesto que de lo contrario se podría llegar a concluir -erróneamente- que la imposibilidad de plantear la violación de un derecho social ante un juez equivaldría a la imposibilidad de hacerlo exigible, lo que no me parece exacto.

\section{Estrategias de exigibilidad de los derechos sociales}

Dicho lo anterior, corresponde ahora considerar algunas posibles líneas estratégicas para hacer exigibles los derechos sociales; es decir, si se acepta en primer lugar que los

2002, pp. 19-20; sobre el concepto de los "derechos a prestaciones", Alexy, Robert, Teoria de los derechos fundamentales, rraducción de Ernesto Garzón Valdés, Madrid, CEPC, 2000 ( $3^{\mathrm{z}}$ reimpresión), pp. 419 y ss.

34 Pisarello, Gerardo, Los derechos sociales y sus garantias. Elementos para una reconstrucción, Madrid, Trotta, 2007, pp. 111 y siguientes. 
derechos sociales no son puras quimeras, y si se acepta también, en segundo término, que del hecho de que algunos de ellos actualmente no se puedan demandar ante un juez por todos sus destinatarios no se desprende la imposibilidad de crear esas vías jurisdiccionales hoy inexistentes, se hace entonces necesario explicitar con cierto grado de detalle todos los argumentos que nos permitan sostener, al contrario de lo que hace la teoría tradicional, una visión normativa plena de los derechos sociales. A ello se dedican las páginas siguientes.

\subsection{El tema de las diferencias estructurales entre derechos sociales $y$ derechos civiles}

Una de las primeras cuestiones que hay que poner en claro para desarrollar la exigibilidad plena de los derechos sociales, es que no existen diferencias estructurales de tal magnitud que hagan completamente diferentes a estos derechos de los derechos civiles y políticos (también llamados derechos de libertad o sencillamente libertades públicas) ${ }^{35}$.

De hecho, aunque es evidente que cada uno de los derechos fundamentales tiene un contenido diverso y despliega en consecuencia efectos normativos diferentes (por ejemplo, no es lo mismo el derecho a la información que la libertad de expresión; de igual manera, no tienen contenidos idénticos la libertad de industria y la libertad de tránsito), no puede decirse que exista algo así como una diferencia genética o estructural entre los derechos sociales y los derechos de libertad.

Contrariamente a lo que podría parecer, también los derechos de libertad requieren, para poder tener relevancia práctica y no quedar como buenos deseos contenidos solamente en el texto de las constituciones, de actuaciones positivas del Estado, las cuales conllevan en no pocas ocasiones importantes erogaciones económicas; conjugan por tanto obligaciones de no hacer y obligaciones de hacer para las autoridades ${ }^{36}$. Lo mismo sucede con los derechos sociales, que generan para la autoridad tanto obligaciones de abstención como obligaciones de realización, que requieren de actividades prestacionales en muchos casos.

Por otro lado, hay sectores de los derechos sociales que entrañan libertades en sentido estricto, como por ejemplo el derecho de huelga o la libertad sindical, que frente a las autoridades generan obligaciones de no hacer, de abstención y de respeto (además de

3s Pisarello, Los derechos sociales y sus garantias, cit., pp. 59 y siguientes.

* Abramovich y Courtis io explican con las siguientes palabras: "....el respeto de derechos tales como el debido proceso, el acceso a la justicia, el derecho a casarse, el derecho de asociación, el derecho de elegir y ser elegido, suponen la creación de las respectivas condiciones institucionales por parte del Estado (existencia y mantenimiento de tribunales, establecimiento de normas y registros que hagan juridicamente relevante la decisión nupcial o el acto de asoxiación, convocatoria a elecciones, organización de un sistema de partidos politicos, etcétera).... (los derechos de libertad) conllevan una intensa actividad estatal destinada a que ouros particulares no interfieran esa libertad y á restablecimiento de la libertad o la reparación del perjuicio una vez producida una intervención indebida, de modo que ral contracara del ejercicio de estos derechos está dada por el cumplimiento de funciones de policia. seguridad, defensa y justicia por parte del Estado", Los derechos sociales como derechos exigibles, cit., pp. 23-24. 
obligaciones de tutela, como en el caso de todos los derechos); en una situación parecida se encuentran aquellos derechos de los trabajadores que no requieren prima facie de prestaciones del Estado para poder ser realizados, como el derecho a un día de descanso semanal, la limitación de la jornada laboral o el derecho a las vacaciones.

No hay, por tanto, "derechos gratuitos" y "derechos caros": todos los derechos tienen un costo y ameritan de una estructura estatal que, al menos, los proteja de las posibles violaciones perpetradas por terceras personas. Podríamos decir, en consecuencia, que un análisis detenido de las categorías "derechos civiles" y "derechos sociales" nos permitiría concluir que no hay elementos suficientes para establecer una división clara entre ellas, $\mathrm{y}$ que las diferencias que pueden existir son más de grado de que sustancia; a partir de esa conclusión se puede defender también la tesis de la indivisibilidad y de la interdependencia de los derechos. Gerardo Pisarello ejemplifica parte de lo que se acaba de decir con las siguientes palabras ${ }^{37}$ :

...todos los derechos fundamentales pueden caracterizarse como pretensiones híbridas frente al poder: positivas y negarivas, en parte costosas y en parte no costosas.

El derecho a la libertad de expresión, en efecto, no sólo supone la ausencia de censura sino también la construcción de centros culturales y plazas públicas, la subvención de publicaciones, la concesión de espacios gratuitos en radios y televisiones o una regulación general que garantice el pluralismo informativo. El derecho de propiedad se garantiza no sólo mediante la ausencia de interferencias estatales arbitrarias sino también mediante la creación de registros inmobiliarios o a través de la financiación estatal de tribunales, jueces y funcionarios que puedan asegurar el cumplimiento de los contratos. El derecho de voto comporta la puesta en marcha de una compleja infraestructura de personal y de material que en ningún caso carece de repercusiones económicas. Incluso el derecho a no ser torturado exige el mantenimiento de centros de detención adecuados y cuerpos policiales formados en principios garantistas.

Del mismo modo, el derecho a la salud no sólo exige el otorgamiento estatal de medicinas gratuitas o a bajo precio sino también la no contaminación de un río o la no comercialización de productos alimenticios en mal estado. El derecho al trabajo no sólo comporta el acceso a un empleo digno sino también la prohibición de despidos ilegítimos. El derecho a una vivienda adecuada no sólo supone... la provisión de viviendas de protección oficial sino también el cumplimiento de otras obligaciones estatales no necesariamente costosas: desde el reconocimiento de seguridad jurídica en la tenencia o la interdicción de las cláusulas abusivas en los contratos de alquiler, hasta

Pisarello, Gerardo, Vivienda para todos: un derecho en (de)construcción. El derecho a una vivienda digna y adecuada como derecho exigible, Barcelona, Icaria, 2003, pp. 29-30. 
la derogación de preceptos discriminatorios en las leyes urbanísticas o la prohibición de desalojos arbitrarios.

Para fundamentar lo dicho en los párrafos anteriores hay que considerar también que, en el caso de algunos derechos sociales, existen derechos de libertad tendientes a proteger bienes jurídicos prácticamente idénticos a los que tutelan aquellos; así por ejemplo, existe una libertad de trabajo, antecedente del derecho social al trabajo; de la misma forma, existe una libertad de educación, indisolublemente vinculada con el derecho social a la educación.

Una vez que se tiene claro lo anterior, no deben existir reticencias hacia el despliegue de efectos normativos plenos y directos de los derechos sociales; estos efectos deben ser exigibles incluso en sede judicial. La teoría constitucional tiene, en este punto, la tarea de pensar en vías idóneas de exigencia de los derechos sociales, sin dejar de tener presente que conllevan efectos presupuestales importantes y que la realización completa de algunos de ellos (vivienda, educación, salud) quizá no pueda darse de forma completa en un plazo corto de tiempo, como es obvio. Pero lo anterior no obsta para señalar con rotundidad que los derechos sociales obligan, que no son buenos deseos o programas políticos, sino simplemente normas jurídicas y que como tales deben ser vistos, analizados y aplicados.

A partir de relativizar las diferencias entre derechos civiles y derechos sociales, como ya se apuntaba, se puede desarrollar el principio de interdependencia e indivisibilidad de los derechos, que fue plenamente reconocido por la Declaración y Programa de Viena, aprobado por la Conferencia Mundial que se llevó a cabo en esa ciudad en 1993; en el punto I.5 de dicho documento se afirma que

"Todos los derechos son universales, indivisibles e interdependientes y están relacionados entre sí. La comunidad internacional debe tratar los derechos humanos en forma global y de manera justa y equitativa, en pie de igualdad y dándoles a todos el mismo peso. Debe tenerse en cuenta la importancia de las particularidades nacionales y regionales, así como de los diversos patrimonios históricos, culturales y religiosos, pero los Estados tienen el deber, sean cuales fueren sus sistemas políticos, económicos y culturales, de promover $y$ proteger todos los derechos humanos y libertades fundamentales".

Todo lo anterior no obsta para reconocer que los derechos sociales tienen un indudable componente prestacional, pues suponen la necesidad de que el Estado lleve a cabo un despliegue importante de actuaciones, muchas de ellas de carácter administrativo, para hacer efectivos los mandamientos relacionados con esos derechos.

¿Qué significa que los derechos sociales sean, en parte, derechos a prestaciones? Robert Alexy lo explica de la siguiente manera: "Los derechos a prestaciones en sentido estricto son derechos del individuo frente al Estado a algo que -si el individuo poseyera medios financieros suficientes y si encontrase en el mercado una oferta suficiente- podria obtenerlo también de particulares. Cuando se habla de derechos sociales fundamentales, 
por ejemplo, del derecho a la previsión, al trabajo, la vivienda y la educación, se hace primariamente referencia a derechos a prestaciones en sentido estricto" ${ }^{18}$.

Las prestaciones a las que hace referencia Alexy no son más que actuaciones del Estado (en forma de bienes y servicios) constatables y medibles, como lo pueden ser la construcción de hospitales, la provisión de equipamientos escolares, la creación de un sistema de pensiones para jubilados, un sistema de sanidad público, la construcción de viviendas o el financiamiento para adquirirlas, etcétera.

Se podría decir, en otras palabras, que los derechos sociales se regulan constitucionalmente como mandatos de optimización, puesto que postulan la necesidad de alcanzar ciertos fines, pero dejan de alguna manera abiertas las vías para lograrlos. Los mandatos de optimización son normas jurídicas redactadas en forma de principios, los cuales, según Alexy, "están caracterizados por el hecho de que pueden ser cumplidos en diferente grado y que la medida debida de su cumplimiento no sólo depende de las posibilidades reales sino también de las jurídicas"; los principios, en opinión de este autor, "ordenan que algo sea realizado en la mayor medida posible, dentro de las posibilidades jurídicas y reales existentes"39.

La obligación de suministrar prestaciones vincula a todos los poderes, no simplemente a las autoridades de carácter administrativo. Desde luego, obligan también al legislador, como lo veremos enseguida.

\subsection{Las obligaciones del Estado en materia de derechos sociales}

En virtud de que aspectos sustanciales de los derechos sociales se satisfacen a través de prestaciones a cargo del Estado, es necesario examinar cuáles son en concreto las obligaciones por medio de las cuales se cumple con esas prestaciones y si existen o no parámetros normativos que las enmarquen. Esto es importante porque nos ayuda a definir el alcance semántico de los derechos sociales, lo cual es una condición para poder desarrollar la plena exigibilidad de los mismos.

\subsubsection{Obligaciones generales}

Tradicionalmente se ha considerado que las obligaciones del Estado en materia de derechos sociales (o incluso, en términos más generales, en relación a todos los derechos fundamentales) tienen tres diversos niveles: respetar, proteger y cumplir o realizar ${ }^{10}$.

La obligación de respetar significa que el Estado -lo que incluye a todos sus organismos y agentes, sea cual sea el nivel de gobierno en el que se encuentren y sea cual sea la forma

\footnotetext{
Alexy, Robert, Teoria de los derechos fundamentales, cit, p. 482.

Alexy, Robert, Teoria de los derechos fundamentales, cit., p. 86.

40 Eide, Absjon, "Realización de los derechos económicos y sociales. Estrategia del nivel mínimo", Revista de la Comisión Internacional de Juristas, número 43, Ginebra, diciembre de 1989, p. 48.
} 
de organización administrativa que adopten- debe abstenerse de hacer cualquier cosa que viole la integridad de los individuos, de los grupos sociales o ponga en riesgo sus libertades y derechos; lo anterior incluye el respeto del Estado hacia el uso de los recursos disponibles para que los sujetos de los derechos puedan satisfacer estos derechos por los medios que consideren más adecuados.

La obligación de proteger significa que el Estado debe adoptar medidas destinadas a evitar que otros agentes o sujetos violen los derechos sociales, lo que incluye mecanismos no solamente reactivos frente a las violaciones (como lo podría ser la creación de procesos jurisdiccionales o sistemas de tutela administrativa), sino también esquemas de carácter preventivo que eviten que agentes privados puedan hacerse con el control de los recursos necesarios para la realización de un derecho.

La obligación de cumplir o realizar significa que el Estado debe adoptar medidas activas, incluso acciones positivas en favor de grupos vulnerables, para que todos los sujetos de los derechos tengan la oportunidad de disfrutar de ellos cuando no puedan hacerlo por sí mismos.

Las obligaciones de los poderes públicos en materia de derechos sociales que genéricamente se acaban de describir han sido detalladas por el Comité de Derechos Económicos, Sociales y Culturales de la ONU en su Observación General número 3, referida justamente a la índole de las obligaciones de los Estados, dictada en su Quinto Periodo de Sesiones, en el año de $1990^{41}$.

La mencionada Observación toma como punto de partida el texto del artículo 2.1. del Pacto Internacional de Derechos Económicos, Sociales y Culturales que establece lo siguiente: "Cada uno de los Estados Partes en el Presente Pacto se compromete a adoptar medidas, tanto por separado como mediante la asistencia y la cooperación internacionales, especialmente económicas y técnicas, hasta el máximo de los recursos de que disponga, para lograr progresivamente, por todos los medios apropiados, inclusive en particular la adopción de medidas legislativas, la plena efectividad de los derechos aquí reconocidos".

Aunque en su conjunto es del máximo interés, del artículo transcrito conviene preliminarmente subrayar tres expresiones: a) todo Estado Parte "se compromete a tomar medidas... por todos los medios apropiados"; b) "hasta el máximo de los recursos de que disponga"; y c) "para lograr progresivamente".

A partir del texto del artículo 2.1. el Comité afirma que el Pacto genera para los Estados Partes tanto obligaciones de comportamiento como obligaciones de resultado; es decir, no se trata de que los Estados deban solamente conducirse de cierta manera, sino también de que logren ciertos objetivos, que se propongan metas y las realicen ${ }^{42}$.

41 Consultable en Carbonell, Miguel, Moguel, Sandra y Pérez Portilla, Karla (compiladores), Derecho Internacional de los Derechos Humanos. Textos Básicos, $2^{2}$ edición, México, CNDH, Porrúa, 2003, tomo 1, pp. 497 y ss.

42 Eide, Absjorn, "Realización de los derechos económicos y sociales. Estrategia del nivel mínimo", p. 48. 


\subsubsection{Tomar medidas adecuadas}

En primer lugar, los Estados deben garantizar el goce de los derechos establecidos en el Pacto sin discriminación alguna, como lo reitera el mismo artículo 2 del Pacto, en su apartado 2. Esta obligación es inmediata y no puede estar sujeta a ningún tipo de limitación u obstáculo (párrafo 1 de la Observación General número 3).

En segundo término, los Estados deben "adoptar medidas apropiadas"; esta obligación debe ser cumplida dentro de un plazo razonablemente corto tras la suscripción del Pacto, con independencia de que la plena realización de todos los derechos pueda llevar un tiempo más prolongado (párrafo 2). Sobre esta obligación Courtis y Abramovich señalan que no es declamativa: "significa que el Estado tiene marcado un claro rumbo y debe comenzar a 'dar pasos', que sus pasos deben apuntar hacia la meta establecida y debe marchar hacia esa meta tan rápido como le sea posible. En todo caso le corresponderá justificar por qué no ha marchado, por qué ha ido hacia otro lado o retrocedido, o por qué no ha marchado más rápido" ${ }^{\not 3}$.

Entre las primeras medidas a tomar se encuentran las de carácter legislativo, lo cual supone fundamentalmente dos cuestiones: la primera consiste en recoger en el ordenamiento jurídico interno todos los derechos que establece el Pacto, de forma que no quede duda sobre su vigencia dentro del territorio del Estado Parte; la segunda consiste en adecuar el ordenamiento interno para el efecto de eliminar cualquier norma que sea contraria a esos derechos o que pueda suponer un obstáculo para su completa realización.

Hay que enfatizar el hecho de que la legislación nacional no solo debe ser no contradictoria con los instrumentos jurídicos internacionales, sino que debe contener las disposiciones necesarias para hacer de ellos normas completamente aplicables por las autoridades locales.

El Comité subraya el hecho de que, en el caso de varios derechos, la existencia de legislación interna es indispensable (párrafo 3). En otra de sus Observaciones Generales, el Comité señala que "los Estados deben modificar el ordenamiento jurídico interno en la medida necesaria para dar efectividad a las obligaciones dimanantes de los tratados en los que sean Parte" 4 .

Otra medida que los Estados parte pueden acometer de inmediato es la de realizar un "diagnóstico" de la situación que guardan cada uno de los derechos protegidos por el Pacto. A partir de ese diagnóstico, los Estados deben elaborar una estrategia nacional para el desarrollo, promoción y protección de los derechos. Entre los objetivos del diagnóstico deben estar el de determinar la proporción de ciudadanos que no disfrutan

43 Abramovich, Victor y Courtis, Christian, Los derechos sociales como derechos exigibles, cit., pp. 79-80.

4 Se trata de la Observación General número 9, relativa a la aplicación interna del Pacto, dictada durante el $19^{\circ}$ periodo de sesiones, en el año de 1998; la cita está en el párrafo 3; esta Observación General es importante porque viene a complementar y, en ciertos aspectos, incluso a detallar el contenido de la Observación General número 3. La número 9 es consultable en Carbonell, Miguel, Moguel, Sandra y Pérez Portilla, Karla (compiladores), Derecho Internacional de los Derechos Humanos. Textos Básicos, tomo I, cit., pp. 550 y ss. 
de un derecho específico y la identificación de los sectores de la población que podrían ser vulnerables o desaventajados para su disfrute ${ }^{45}$.

El diagnóstico debe poder ofrecer una serie de instrumentos de medición que sirvan tanto al Estado como al Comité para medir concretamente si se ha avanzado o no en la consecución de un derecho determinado. Los instrumentos de medición son determinados sobre una base y unos criterios nacionales, lo cual sirve para poner a salvo la gran variedad de contextos económicos y sociales existentes, objetivo que no se lograría bajo un solo nivel de medición determinado internacionalmente. En contra de esta consideración se ha dicho que con ella se podría vaciar el significado de muchos contenidos sustantivos del Pacto ${ }^{46}$. En realidad quizá lo mejor sería que la determinación nacional se mueva dentro de ciertos parámetros, de manera que no se deje una completa discrecionalidad a los Estados. Como quiera que sea, el Comité ha sostenido que "los medios utilizados deben ser apropiados en el sentido de producir resultados coherentes con el pleno cumplimiento de las obligaciones por el Estado Parte" ${ }^{\$ 7}$.

Junto a las medidas legislativas y de diagnóstico deben adoptarse también, en virtud del mandato de utilizar "todos los medios apropiados", previsiones de carácter administrativo, judicial, económico, social y educativo. En este sentido, hay que decir que en algunas disposiciones del Pacto pueden encontrarse medidas concretas que los Estados deben tomar para implementarlo. Por ejemplo, en el artículo 13.2. se establece que la enseñanza primaria debe ser obligatoria y gratuita para todos como medida para realizar el derecho a la educación. El Comité ha recalcado que aunque en el texto del Pacto se utilicen supuestos ejemplificativos o ilustrativos (como en los artículos 6.2, 11.2, 12.2, 13.2 o 15.2), no por ello dejan de ser obligatorios ${ }^{48}$.

En principio, cada Estado Parte debe determinar por sí mismo cuáles son las medidas más apropiadas que debe tomar para cumplir con las obligaciones del Pacto, considerando sus propias circunstancias y la relación de todos los derechos protegidos. Sin embargo, la "propiedad" de las medidas puede no resultar evidente a primera vista, por lo cual los Estados deben informar claramente al Comité, en términos del artículo 16 del propio Pacto, por qué consideran que las medidas adoptadas son las más pertinentes en vista de las circunstancias (párrafo 4).

\subsubsection{Creación de recursos legales para defender los derechos sociales}

Aparte de las medidas que ya se han comentado, el Comité considera concretamente que una medida apropiada consiste en el ofrecimiento de recursos judiciales para proteger

45 En este sentido, Craven, Matthew, The international covenant on economic, social and cultural rights. A perspective on its development, Oxford, Clarendon Press, 1995, p. 117.

46 Craven, Matthew, The international covenant on economic, social and cultural rights. A perspective on its development, cit., p. 119.

47 Observación General número 9, párrafo 5.

48 Craven, Marthew, The international covenant on economic, social and cultural rights. A perspective on its development, cit., p. 116. 
los derechos, de forma que todos ellos puedan considerarse justiciables (párrafo 5). En la Observación General número 9 el Comité ha explorado con mayor detenimiento esta obligación de los Estados Parte.

En esta Observación el Comité reconoce que no se trata solamente de crear recursos judiciales, sino de implementar un concepto más amplio al que denomina "recursos legales". Dentro de esos recursos se encuentran también los judiciales, pero no son los únicos ya que el Comité reconoce que los recursos administrativos "en muchos casos son adecuados", ya que "quienes viven bajo la jurisdicción de un Estado Parte tienen la expectativa legítima de que, sobre la base del principio de buena fe, todas las autoridades administrativas, al adoptar decisiones, tendrán en cuenta las disposiciones del Pacto". Los recursos administrativos, no obstante, deben reunir ciertas características, como por ejemplo ser accesibles, no onerosos, rápidos y eficaces; en cualquier caso, debe existir la posibilidad de plantear una apelación judicial contra todo proceso administrativo (OG número 9, párrafo 9).

El Comité distingue entre el concepto de justiciabilidad de los derechos sociales y el concepto de "aplicabilidad inmediata". El primero se refiere a las cuestiones que pueden o deben resolver los tribunales; a lo anterior hay que agregar que la justiciabilidad también significa que los individuos y los grupos tengan la posibilidad de acudir ante esos mismos tribunales, lo cual es una pre-condición para luego estar en aptitud de determinar el ámbito en el que la decisión judicial es pertinente. Para el Comité la aplicabilidad inmediata de un derecho significa que ese derecho permite la aplicación por los tribunales sin mayor disquisición. El Comité señala que todos los derechos reconocidos en el Pacto tienen, al menos en algún aspecto, dimensiones significativas que puedan ser llevadas ante los tribunales, es decir, dimensiones de justiciabilidad (OG número 9, párrafo 10).

Respecto de la aplicabilidad inmediata el Comité realiza una consideración importante en los siguientes términos:

A veces se ha sugerido que las cuestiones que suponen una asignación de recursos deben remitirse a las autoridades políticas y no a los tribunales. Aunque haya que respetar las competencias respectivas de los diversos poderes, es conveniente reconocer que los tribunales ya intervienen generalmente en una gama considerable de cuestiones que tienen consecuencias importantes para los recursos disponibles. La adopción de una clasificación rígida de los derechos económicos, sociales y culturales que los sitúe, por definición, fuera del ámbito de los tribunales sería, por lo tanto, arbitraria e incompatible con el principio de que los dos grupos de derechos son indivisibles e interdependientes. También se reduciría drásticamente la capacidad de los tribunales para proteger los derechos de los grupos más vulnerables y desfavorecidos de la sociedad (OG número 9, párrafo 10). 
Sobre el tema de la existencia de recursos judiciales que permitan llevar ante los tribunales las violaciones de los derechos sociales reconocidos en el Pacto, Abramovich y Courtis señalan que los Estados deben brindar recursos judiciales idóneos; es decir, "no basta con los recursos previstos para reparar la violación de otros derechos, cuando por sus características impidan el planteo adecuado del caso" ${ }^{\prime 29}$. Es muy relevante enfatizar la idoneidad de los recursos judiciales existentes, ya que no puede considerarse como cumplida por los Estados Parte la obligación de brindar esos recursos por el simple hecho de que se aduzca que existen las vías judiciales tradicionales en caso de violación de derechos; hay que considerar que en la enorme mayoría de países los recursos judiciales existentes fueron diseñados para proteger derechos civiles y políticos, por lo que tienen enormes problemas al momento en que se les pide que sirvan para proteger derechos sociales.

El Comité seńala que para la mejor aplicación del Pacto a nivel interno es importante informar a los jueces y a los tribunales competentes de la naturaleza y las consecuencias del propio Pacto, así como explicarles la importante función que desempeñan los recursos judiciales en su aplicación (OG número 9, párrafo 11). Esto es muy importante, sobre todo porque en muchos países los tribunales en términos generales desconocen los contenidos del Pacto (lo mismo sucede, por otro lado, con los abogados), lo que genera que en la práctica se aplique escasamente. Reconociendo esa situación el Comité afirma que "En la mayoría de países, los tribunales todavía están lejos de recurrir suficientemente a las disposiciones del Pacto" (OG número 9, párrafo 13).

El texto del Pacto, reconoce el Comité, puede aplicarse dentro de una amplia variedad de sistemas políticos y económicos, de forma que no está condicionada la protección de los derechos a la adopción de uno de ellos, siempre que queden adecuadamente reconocidos y estén reflejados en el sistema de que se trate (OG 3, párrafo 8).

Para cumplir con la obligación de crear recursos legales que permitan defender los derechos sociales se tendrían que ampliar los cauces para promover acciones ante los tribunales, para lo cual también sería necesario dotar de sustantividad procesal a los denominados "derechos difusos" o "intereses colectivos" $"$. A partir del reconocimiento de dicha sustantividad procesal (pues es obvio que derechos como el medio ambiente o en ciertos aspectos el derecho a la educación y a la salud son materialmente difusos y protegen intereses colectivos), habría que ir modelando las estrategias de defensa procesal necesarias para cumplir con los señalamientos del Comité en materia de defensa de los derechos sociales. Este aspecto es especialmente importante en México, ya que el principal instrumento de defensa jurisdiccional de los derechos fundamentales, que es el juicio de amparo, se ha mostrado insuficiente para proteger derechos sociales justamente en virtud de la estrecha legitimación activa que tanto la Constitución como la ley y la jurisprudencia reconocen para promoverlo ${ }^{51}$.

49 Abramovich, Victor y Courtis, Christian, Los derechos sociales como derechos exigibles, cit., p. 87.

s9 Para una introducción al tema ver Gidi, Antonio y Ferrer MacGregor, Eduardo (coordinadores), La tutela de los derechos difusos, colectivos e individuales homogéneos, México, Porrúa, 2003.

si Ver las reflexiones en el mismo sentido de Ferrer MacGregor, Eduardo, Juicio de amparo e interés legitimo: la tutela de los derechos difusos y colectivos, México, Porrúa, 2003. 


\subsubsection{Obligación de progresividad y prohibición de regresividad}

La obligación de "lograr progresivamente... la plena efectividad de los derechos reconocidos" implica el reconocimiento del hecho de que la plena efectividad de todos los derechos económicos, sociales y culturales no podrá lograrse en un periodo breve de tiempo (OG número 3, párrafo 9).

Pero lo anterior no significa que se prive a la obligación contenida en el artículo 2.1. de todo contenido significativo. Por el contrario, la obligación de progresividad significa antes que nada que los esfuerzos en la materia deben darse de forma continuada, con la mayor rapidez y eficacia que sea posible alcanzar, de manera que se logre una "mejora continua de las condiciones de existencia", como lo ordena el artículo 11 del Pacto.

De la obligación de progresividad se desprende también la prohibición de regresividad, es decir, la prohibición de que los Estados Parte den marcha atrás en los niveles alcanzados de satisfacción de los derechos; por eso se puede afirmar que la obligación de los Estados Parte en relación con los derechos establecidos en el Pacto es de carácter ampliatorio, "de modo que la derogación o reducción de los derechos vigentes contradice claramente el compromiso internacional asumido" 52 .

Sobre este punto el Comité señala que "todas las medidas de carácter deliberadamente restrictivo en este aspecto deberán justificarse plenamente por referencia a la totalidad de los derechos previstos en el Pacto y en el contexto del aprovechamiento pleno del máximo de los recursos de que se disponga" (OG número 3, párrafo 9).

Abramovich y Courtis han señalado que la obligación de progresividad constituye un parámetro para enjuiciar las medidas adoptadas por los poderes legislativo y ejecutivo en relación con los derechos sociales, es decir, se trata de una forma de carácter sustantivo a través de la cual los tribunales pueden llegar a determinar la inconstitucionalidad de ciertas medidas (o al menos su ilegitimidad a la luz del Pacto) ${ }^{53}$.

Toda medida regresiva se presume violatoria del Pacto y al Estado corresponde la carga de la prueba para demostrar que no lo es o que, siendo regresiva, está justificada ${ }^{54}$. Para poder justificar una medida regresiva el Estado tendrá que demostrar ${ }^{55}$ : a) la existencia de un interés estatal permisible que la medida regresiva tutela; b) el carácter imperioso de la medida; yc) la inexistencia de cursos de acción alternativos que pudieran ser menos restrictivos del derecho que se haya visto afectado de forma regresiva.

Desde luego, si la medida regresiva está dirigida a excluir de los niveles mínimos de protección a ciertas personas, entonces se considera que viola el Pacto, sin que el Estado pueda justificar en forma alguna esa medida ${ }^{56}$.

\footnotetext{
Abramovich, Victor y Courtis, Christian, Los derechos sociales como derechos exigibles, cit., p. 94. Ver también Pisarello, Los derechos sociales y sus garantias, cit., pp. 64-66.

Abramovich, Victor y Courtis, Christian, Los derechos sociales como derechos exigibles, cit., p. 95.

4 Abramovich, Víctor y Courtis, Christian, Los derechos sociales como derechos exigibles, cit., p. 105.

Abramovich, Vicror y Courtis, Christian, Los derechos sociales como derechos exigibles, cit., p. 109.

s6 Abramovich, Victor y Courtis, Christian, Los derechos sociales como derechos exigibles, cit., p. 110.
} 


\subsubsection{Destinar el máximo de recursos disponibles}

La obligación establecida en el artículo 2.1. del Pacto, consistente en destinar "el máximo de los recursos de que disponga" a la realización de los derechos establecidos en el propio Pacto, significa que los recursos del Estado, así sean insuficientes para la satisfacción completa de un derecho, deben ser empleados para dar cumplimiento al contenido del Pacto.

La misma obligación genera para los Estados Parte la carga de la prueba a fin de demostrar que en efecto se han empleado "todos los recursos disponibles" para cumplir con los objetivos del Pacto en el plazo más breve posible. Es decir, la falta o insuficiencia de recursos no debe tomarse como una verdad prima facie, sino que debe ser acreditada por el Estado.

La obligación de informar y de transparentar la gestión pública es muy importante para el caso de todos los derechos sociales, especialmente en derechos como la salud o la vivienda que involucran un monto considerable de recursos y que suponen grandes zonas de opacidad en el funcionamiento de la administración pública.

\subsubsection{Periodos de crisis y niveles mínimos de los derechos sociales}

Para efecto de cumplir con la obligación de destinar el máximo de los recursos disponibles no obsta el hecho de que un país se encuentre en periodo de "ajuste estructural" o pasando por una crisis económica, pues aparte de que los derechos fundamentales están vigentes en todo tiempo, el Comité se ha encargado de enfatizar que es justamente en tiempos de contracción económica cuando mayor relieve toman las obligaciones estatales para satisfacer los derechos y cuando más atención se debe poner a la correcta utilización de los recursos disponibles.

En periodos de crisis los Estados, cuando menos, deben asegurar las mejores condiciones posibles para los grupos más desaventajados": "aun en tiempos de limitaciones graves de recursos -afirma el Comité- causadas por el proceso de ajuste, de recesión económica o por otros factores, se puede y se debe en realidad proteger a los miembros vulnerables de la sociedad mediante la adopción de programas de relativo bajo costo" (OG número 3, párrafo 12).

Junto a lo anterior, los Estados Parte deben también estar en condiciones de ofrecer unos niveles minimos de satisfacción de los derechos. El Comité ha señalado que "Si el Pacto se ha de interpretar de tal manera que no establezca una obligación mínima, carecería en gran medida de su razón de ser" (OG número 3, párrafo 10).

Si en un Estado Parte no se pudieran satisfacer esos niveles mínimos se estaría frente a una "presunción de culpabilidad" del Estado en el incumplimiento de las disposiciones del Pacto; esta presunción puede ser derrotada si el Estado en cuestión demuestra que

57 Craven, Mathew, The international covenant on economic, social and cultural rights. A perpective on its development, cit., p. 139. 
la situación de crisis ha sido de tal magnitud que ha estado fuera de su control y de sus posibilidades la satisfacción de los niveles mínimos en materia de derechos sociales.

En palabras del Comité, "Para que cada Estado Parte pueda atribuir su falta de cumplimiento de las obligaciones mínimas a una falta de recursos disponibles, debe demostrar que ha realizado todo esfuerzo para utilizar todos los recursos que están a su disposición en un esfuerzo por satisfacer, con carácter prioritario, esas obligaciones mínimas" (párrafo 10).

Craven señala, sin embargo, que dichos niveles mínimos no han sido determinados por el Comité, por lo cual no se tienen los instrumentos que permitan medir con precisión pero con flexibilidad los eventuales incumplimientos de los Estados parte ${ }^{58}$. Al respecto, Abramovich y Courtis señalan que

Si bien la tarea del Comité puede fijar los contenidos esenciales que identifiquen a ciertos derechos, resulta claro que tal propósito no puede alcanzarse sólo con volcar conceptos jurídicos en un texto. Numerosas opiniones han propuesto la adopción de algún sistema de indicadores que pudiera servir como parámetro. La utilización de indicadores resulta especialmente relevante cuando se exige al Estado el cumplimiento de ciertos objetivos que resultan mesurables, como la erradicación del analfabetismo, el tratamiento de las enfermedades endémico-epidémicas, la reducción de la morti-natalidad infantil o la siniestralidad laboral. En los últimos ańos, además, se ha comenzado a trabajar en la correlación entre la noción de contenido mínimo esencial de un derecho y los parámetros de desarrollo social y otros estándares técnicos establecidos a partir de indicadores estandarizados a nivel mundial. El principal propósito de todo sistema de indicadores es dar cuenta de dos factores claves, la voluntad y la capacidad del Estado de promover y proteger los derechos humanos. La diferencia entre estos dos factores, particularmente en relación a los derechos económicos, sociales y culturales, es crucial para examinar el comportamiento del Estado 59 .

En relación al contenido mínimo, es importante seńalar que se trata de un concepto aplicable no solamente a los derechos sino también a sectores de la población; concretamente, se puede aplicar para identificar al mínimo de personas a las que el Estado debe proteger en caso de crisis económica. Es decir, el contenido mínimo en relación a grupos obliga al Estado a identificar a las personas en situación de vulnerabilidad para asegurarles el disfrute de sus derechos sociales; por ejemplo en materia de derecho a la vivienda se considera como grupo vulnerable a los ancianos sin recursos y a las personas con discapacidad; en relación a ellos el Estado debe suministrar una protección especial ${ }^{60}$.

58 Craven, Matthew, The international covenant on economic, social and cultural rights. A perspective on its development, cit., p. 143.

59 Abramovich, Victor y Courtis, Christian, Los derechos sociales como derechos exigibles, cit., pp. 91-92.

wo Abramovich, Victor y Courtis, Christian, Los derechos sociales como derechos exigibles, cit., p. 92. 
Como señala Eide, "El umbral mínimo para enfocar el problema sostiene que el establecimiento de un nivel mínimo de satisfacción de necesidades es un requisito previo esencial de esta consecución progresiva de la realización de los derechos. La justicia distributiva de largo curso para realizar las normas completas de los derechos humanos requiere la justicia inmediata para aquellos grupos de personas más desfavorecidos"61.

\section{Conclusión}

Si quisiéramos intentar resumir las principales ideas que tendría que incorporar una teoría constitucional comprometida con la eficacia de los derechos sociales, quizá tendríamos que subrayar las siguientes obligaciones a cargo de los poderes públicos (las cuales, por otro lado, sintetizan lo que se ha dicho en los apartados anteriores):

a) Tutelar los derechos sin discriminación.

b) Tomar todas las medidas apropiadas para hacer efectivos los derechos dentro de su territorio.

c) Demostrar que las medidas tomadas son las más apropiadas para alcanzar los objetivos del Pacto.

d) Establecer vías judiciales para llevar ante los tribunales las posibles violaciones a los derechos señalados.

e) Lograr progresivamente la satisfacción de los derechos establecidos en el texto constitucional y en los tratados internacionales, entendiendo por progresividad la obligación de hacerlo de manera inmediata y continua.

f) No dar marcha atrás en los niveles de realización alcanzados, puesto que está prohibida o severamente restringida la regresividad.

g) Destinar el máximo de recursos disponibles a cumplir con los derechos sociales fundamentales.

h) Acreditar que en efecto se ha destinado el máximo de recursos disponibles.

i) En periodos de crisis, priorizar la protección de los miembros más vulnerables de la sociedad; $y$

j) Asegurar niveles mínimos de satisfacción de los derechos, los cuales deben ser mantenidos incluso en periodos de crisis o de ajustes estructurales.

6) Eide, Absjorn, "Realización de los derechos económicos y sociales. Estrategia del nivel mínimo", p. 54. 
